\title{
Continuidad entre la ciencia y el arte en el movimiento Zapatista: una mirada desde la complejidad
}

\author{
Francisco De Parres Gómez
}

\begin{abstract}
Resumen
Como una de las perspectivas más actuales de la ciencia, existen posicionamientos que invitan a romper con las barreras disciplinares; lo anterior se plantea como una necesidad para solucionar infinidad de problemas que enfrentamos de manera conjunta como sociedad en crisis y donde es preferible que los científicos ya no se posicionen en un solo campo de conocimiento. Así, desde la perspectiva de la complejidad, se propone que es necesario tender puentes para unir desde los conocimientos más abstractos como las matemáticas y la física, pasando por la antropología o la historia, hasta conocimientos prácticos y sensibles como los que nos propone el arte; sin embargo, como exploraremos en el texto, este tipo de propuestas no sólo pertenecen a la academia, sino que también se están retomando como parte de algunos movimientos sociales de avanzada en México, en especial por el Ejército Zapatista de Liberación Nacional (EZLN).
\end{abstract}

Palabras clave: complejidad, ciencia, arte, zapatismo, movimientos sociales.

\section{Continuity between sCience and art in the Zapatista movement: A LOOK FROM THE COMPLEXITY}

\begin{abstract}
As one of the most current perspectives of science, there are positions that invite us to break with disciplinary barriers, which is considered as a necessity in order to solve the countless problems that we face together as a society in crisis and where it is preferable that scientists no longer position themselves in a single field of knowledge; In this context, Complexity perspective proposes the necessity to build bridges to unite from more abstract knowledge such as mathematics and physics, through anthropology or history, to practical and sensitive knowledge such as those proposed by art; However, as we will explore in this paper, this kinds of proposals not only belong to the academy, but have also being reconsidered by some advanced social movements in Mexico, especially by the Zapatista Army of National Liberation.
\end{abstract}

Key words: complexity, science, art, zapatism, social movements. 


\section{Francisco De Parres Gómez}

Maestro en Antropología Social y licenciado en Comunicación Social. Es miembro de la Asociación de Antropólogos Iberoamericanos en Red (España), de la Unión de Científicos Comprometidos con la Sociedad (México) y del Padrón Nacional de Jóvenes Investigadores (UNAM). Cofundador del Colectivo Transdisciplinario de Investigaciones Críticas (COTRIC). Premiado por el Programa de Estímulo a la Creación y Desarrollo Artístico organizado por el Instituto Veracruzano de la Cultura y Consejo Nacional para la Cultura y las Artes. Ponente en foros nacionales e internacionales. Autor de varios capítulos de libros. En la actualidad cursa el doctorado en Antropología Social en la Escuela Nacional de Antropología e Historia, en la línea de investigación Análisis del discurso y Semiótica de la cultura, donde desarrolla una investigación dentro de las áreas de interés que ya ha venido trabajando como: las estéticas de la ruptura, el binomio arte-resistencia, los movimientos sociales, la estética decolonial, entre otros.

\section{Sobre los fundamentos de la ciencia moderna}

Divide las dificultades que examinas en tantas partes como sea posible para su mejor solución.

René Descartes

Dentro del desarrollo y construcción de lo que se conoce como ciencia, la humanidad siempre se ha caracterizado por estar continuamente en la búsqueda de respuestas que nos ayuden a comprender mejor al mundo en el que vivimos; es así que, como parte de este proceso, han existido ideas que marcaron profundamente los distintos periodos de la historia; por ejemplo, las investigaciones realizadas por los pensadores del periodo del Renacimiento del siglo XVII y la Ilustración a mediados del siglo XVIII en Europa, entre los que destaca René Descartes, considerado como uno de los principales fundadores de la ciencia moderna.

Si bien, en su momento, las propuestas que trataban de establecer las características del método científico produjeron amplios e interesantes debates, parte de las críticas que se pueden hacer hoy a esta forma de producir conocimiento (que en algunas investigaciones se sigue utilizando), es que se basa en entender que existen divisiones entre el cuerpo y la mente, o entre la razón y las emociones.

Lo anterior, para justificar que el modo de actuar de los científicos debe de ser "objetivo" y así hacer investigaciones "verdaderamente científicas" con resultados comprobables; sin embargo, en la realidad más cotidiana podemos observar que esto ocurre de una manera muy distinta, basta observar cómo las personas, cuando se interesan por algún tema en especial, lo hacen porque se sienten atraídos y quieren conocer más, de tal manera que la curiosidad misma ya está cargada de emociones, al menos de aceptación o rechazo. 
Ilustración por Alberto Montt. S/f. Recuperada de: http://www.dosisdiarias.com/ Creative Commons (Consultado el 15 de marzo de 2018). ...los pueblos originarios y quienes viven, resisten y luchan en los sótanos en todo el mundo son poseedores, entre otras, de una sabiduría fundamental: la de la supervivencia en condiciones adversas...
Así, los pensadores que fundaron la ciencia moderna, concebían, a grandes rasgos, que los investigadores debían ser imparciales y no dejarse influir por las emociones, lo que provocó que se establecieran como acciones opuestas el conocimiento científico y a las prácticas que surgen desde lo que sentimos, como el arte. Pero en realidad no advertían que existe una continuidad entre todas estas formas de conocimiento.

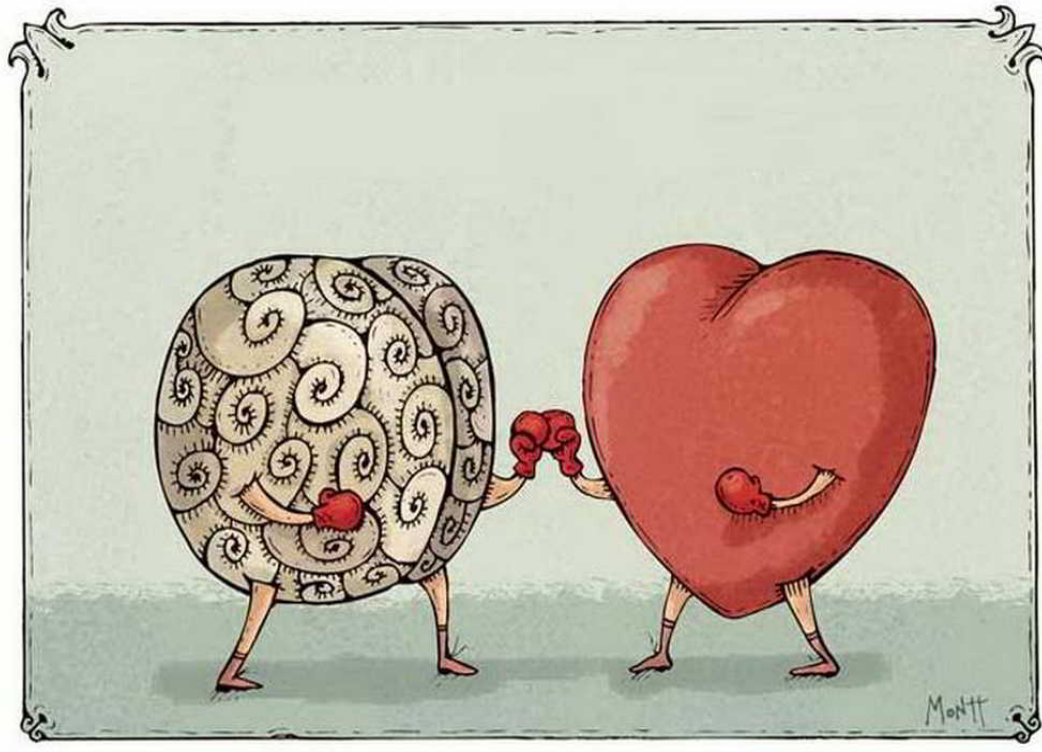

Para entender mejor cómo estaba fundamentado el modelo de la ciencia moderna, se defendía, por ejemplo, que los investigadores se encontraban separados de sus temas de estudio, además de que para ser más exactos en lo que se intentaba descubrir, era necesario que tuvieran un conocimiento altamente especializado y por lo tanto más simplificado y fragmentado, tal como decía Descartes respecto a dividir lo que se quiere estudiar en el mayor número de partes posibles, de tal forma que, si se procede de esta manera, es difícil encontrar diálogos que den cuenta de la complejidad de la vida de manera extensa.

Es pertinente decir que en ese tiempo el conocimiento estaba reservado para las clases ilustradas. Además, estos principios del método científico fueron fundados en Europa, por lo tanto, eran excluyentes tanto a los diferentes estratos de la sociedad como a otras poblaciones que no formaran parte del mediterráneo, con esto se dejaba fuera a toda la sabiduría de otros pueblos que se consideraban atrasados o incivilizados, y así se hicieron aún más grandes las separaciones y las brechas entre las poblaciones del mundo.

En esta misma línea, es necesario apuntar que la realidad es mucho más densa y compleja, empezando por reconocer, ya no desde una posición científica sino de la manera más amplia, como seres humanos, que todos interactuamos constantemente con distintos campos de conocimientos, es decir, ponemos en práctica la transdisciplina pero necesitamos ser conscientes de ello: hacemos uso de la física en nuestros desplazamientos diarios; utilizamos la química cuando cocinamos; nuestro cuerpo tiene procesos biológicos, microcelulares y neurocerebrales sin los cuales no podríamos vivir; ponemos en práctica nuestra inteligencia matemática y económica cuando adquirimos 
algún producto o servicio; al mismo tiempo que pertenecemos a una historia global como parte de un periodo específico en el tiempo; es decir, constantemente en nuestro día a día retomamos muchos terrenos de conocimiento que, en realidad, están más unidos y continuos que separados o sin relación; por lo tanto, para reconocer esa transdisciplinariedad y complejidad tanto de los sujetos como de la vida, surgen nuevos caminos que nos invitan a replantear la ciencia como hasta ahora la hemos conocido.

\section{La complejidad y la transdisciplina como propuestas contemporáneas}

...la complejidad aparece allí donde el pensamiento simplificador falla, pero integra en sí misma todo aquello que pone orden, claridad, distinción, precisión en el conocimiento. Mientras que el pensamiento simplificador desintegra la complejidad de lo real, el pensamiento complejo integra lo más posible los modos simplificadores de pensar...

(Morin, 1997: 3)

Es así que, como una de las propuestas más actuales cuyo objetivo es superar estas separaciones creadas de manera artificial (por la ciencia moderna) dentro de las disciplinas científicas, surge la teoría de la complejidad y la transdisciplina, cuyos principales impulsores son Edgar Morin y Basarab Nicolescu. En consecuencia, desde esta perspectiva se busca integrar el conocimiento de campos que antes se estudiaban de forma separada o simplificada, es decir, se aspira hacia un abordaje complejo de nuestros objetos de estudio, y se deja claro que estas propuestas no deben verse como un pensamiento acabado o delimitado,

ya que se concibe la construcción del conocimiento como un continuo proceso

Imagen recuperada de: https://sociedadcriticaco. wordpress.com/

Creative Commons (Consultado el 15 de marzo de 2018).

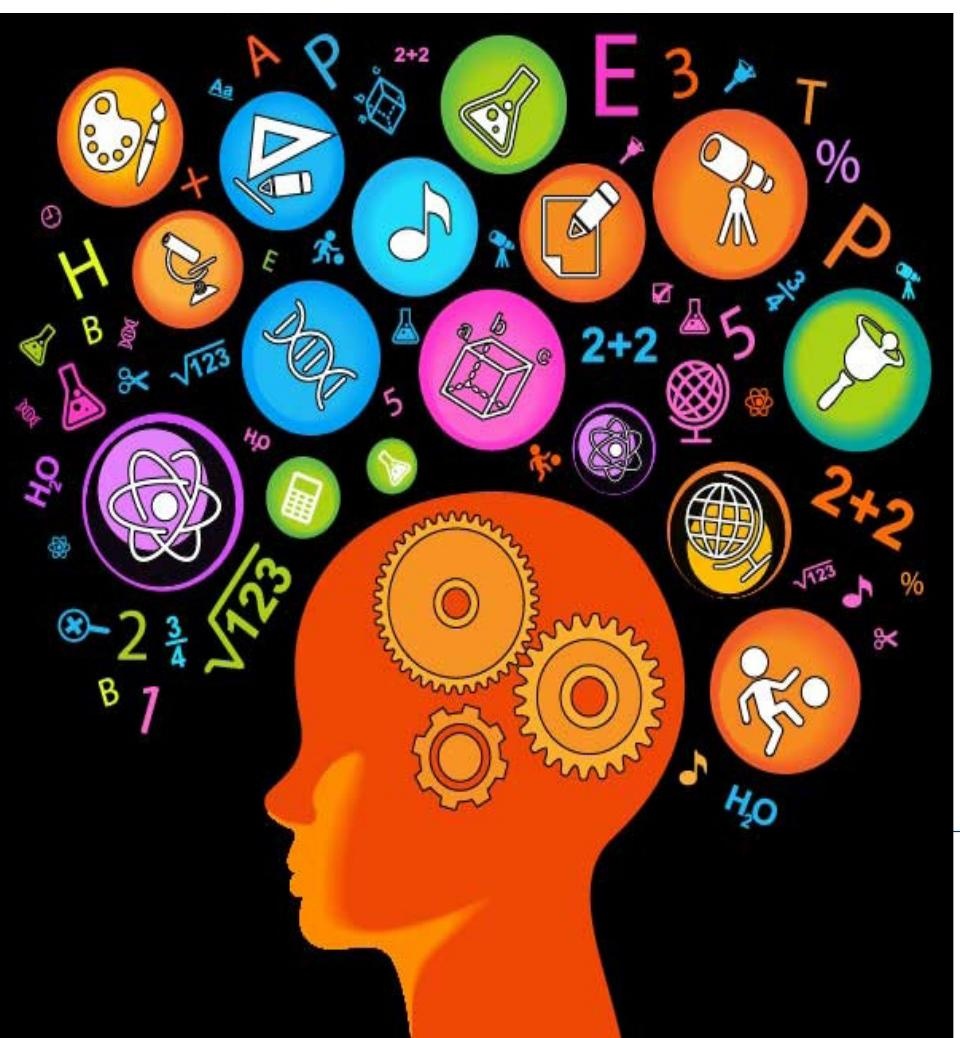

de transformación, a diferencia del pasado donde se defendía una visión acumulativa del conocimiento como si se intentara llegar a tener la última verdad.

¿Pero, qué entendemos cuando hablamos de complejidad y transdisciplina? De forma básica, lo podemos pensar como la construcción de una red que busca unir conocimientos que antes se estudiaban de forma aislada, donde se integran incluso aquellos que previamente se consideraban como excluyentes, por ello hay que mirar la realidad desde una perspectiva que se define como transdimensional, es decir, que la realidad se compone de diferentes dimensiones muy diversas que van desde la social, la psicológica, la económica, la histórica, la política, la del lenguaje, y demás niveles que nos interesen que se relacionan con nuestro tema de estudio y que hay que analizar de manera articulada. 
Como ejemplo, podemos pensar que cuando estudiamos un fenómeno desde una sola disciplina, como tradicionalmente se hacía, es como si alumbráramos con una linterna el objeto que queremos conocer pero sólo alcanzáramos a dar luz a una de sus caras, por lo tanto, necesitamos más linternas, o en nuestro caso, disciplinas y sus herramientas para alumbrar nuestro tema desde el mayor número de ángulos posibles, lo que nos ayudará a comprenderlo mejor.

Desde la perspectiva de la complejidad y la transdisciplina, lo que se planeta es que esta forma de conocer impacte en todos los niveles de la investigación, desde cómo concebimos lo humano y la realidad, nuestra posición frente al cómo conocemos, qué herramientas utilizamos, cómo las aplicamos y hasta los temas que elegimos; por lo tanto, es fundamental en este planteamiento que se logren ampliar las posibilidades de hacer investigación, contribuyendo a una reflexión crítica y sobre todo ética acerca de cómo nuestro trabajo transforma la realidad.

En la actualidad aún existen varias rupturas entre las ciencias naturales, las ciencias sociales, las ciencias cuantitativas y las ciencias artísticas (sólo por mencionar algunas), donde es preciso pensar la necesidad de replantear el conocimiento para tender puentes, transgredir las barreras y encontrar las continuidades desde las ciencias más "duras" hasta el conocimiento que se produce desde el arte, en palabras de Velilla: “...no se trata de suprimir las disciplinas, sino por el contrario de hacerlas aún más útiles, coordinándolas e integrándolas, lo cual implica una educación mental y una estructura de pensamiento capaz de afrontar la complejidad, que es el mejor antídoto contra los pensamientos únicos." (Velilla, 2002:8).

En ese sentido no sería lo mismo complicado que complejo, ya que podríamos definir lo primero como la incapacidad de comprender cierto tema o problema, mientras que lo complejo corresponde a una riqueza de conocimiento que desborda en sí mismo tanto nuestro propio pensamiento como a nuestros temas de estudio.

Uno de los retos más grandes con que se encuentra el planteamiento de la complejidad y la transdisciplina, es que no bastaría con unificar las diferentes ciencias y artes que se producen desde la academia, sino además es necesario integrar otros conocimientos igual de valiosos como los que se dan en la vida cotidiana y fundamentalmente, los que históricamente han estado fuera de los grupos de poder de las universidades o los países que son potencias mundiales, es decir, lo que se produce fuera de los círculos intelectuales y, sobretodo, el conocimiento de los pueblos originarios.

Por ello, como veremos a continuación, además de unificar el conocimiento que se produce desde las diferentes esferas de la vida de manera compleja, es necesario que este se pueda aplicar para mejorar las condiciones de existencia de toda la población a nivel mundial. En ese sentido, actualmente experimentamos periodos de fuerte descomposición del tejido social producto de una inequitativa distribución de la riqueza, que se traduce en un acceso desigual a los 
recursos que posibiliten tener una vida digna; no obstante, si bien existen innumerables movimientos sociales que pugnan por los derechos de los más desfavorecidos, no en todos los casos se hace énfasis en que el factor más importante para salir de la crisis es la continuidad existente entre las ciencias y las artes; sin embargo, en las comunidades autónomas zapatistas del sureste mexicano, se comienza a discutir sobre la urgencia de recuperar estos planteamientos.

En ese sentido, es importante señalar cómo desde el Zapatismo se están articulando conocimientos, por ejemplo, desde la política con la crítica que hacen los movimientos sociales al Estado, la sociología que plantea nuevas formas de organización, la historia que reconoce a los pueblos originarios como sistemáticamente excluidos, la antropología que rescata el valor de los diferentes grupos culturales, el arte como herramienta de denuncia, además de áreas como la agroecología, la medicina, la geografía, la física y demás dimensiones del conocimiento que en articulación todas ellas pueden construir mejores formas de existir de manera global.

\section{Preparando el terreno: practicar la ciencia y el arte para construir un mundo nuevo}

Es nuestra creencia que la posibilidad de un mundo mejor (no perfecto ni acabado, dejemos eso para los dogmas religiosos y políticos) está fuera de la máquina y su posibilidad se sostiene en un trípode. O más bien en la interrelación entre tres columnas que han pervivido y perseverado, con sus altibajos, sus pequeñas victorias y sus grandes derrotas, a lo largo de la breve historia del mundo: las artes...las ciencias y los pueblos originarios con los sótanos de la humanidad.

Subcomandante Insurgente Galeano, 2016

Si bien el movimiento indígena conformado por el Ejército Zapatista de Liberación Nacional (EZLN) ha tenido diferentes momentos de recomposición desde su levantamiento en armas el 1 de enero de 1994; a partir del 21 de diciembre de 2012, cuando más de 50,000 de sus integrantes hicieron una marcha en algunas de las principales cabeceras municipales de Chiapas, en lo que se conoció como la "Marcha del silencio", podemos decir que se dio paso hacia una nueva etapa de vinculación y apertura de los y las zapatistas para con la sociedad mexicana y la internacional (Arcos y Zagato, 2017: 82-85), proceso que iniciaron con esta especie de performance artístico-político, donde sus integrantes marcharon de manera silenciosa en filas que formaban espirales de caracol, para al final todos y cada uno subir a una tribuna y alzar el puño, con lo que simbólicamente representaban que comparten el poder como se ejerce en sus comunidades, es decir, a través del principio zapatista del "mandar-obedeciendo", o en otras palabras donde "el pueblo manda y el gobierno obedece".

Como se puede observar, este acto artístico-político fue una multitudinaria reaparición del EZLN en la vida pública cuando se decía que ya había desaparecido, aunque en realidad sólo no había tenido atención de los medios de comuni- 
Da clic en la imagen para reproducir el video. cación comerciales; con esto se demostró, que se mantuvieron reorganizándose al interior de sus comunidades para construir su autonomía en colectividad (Ibídem: 83-84).

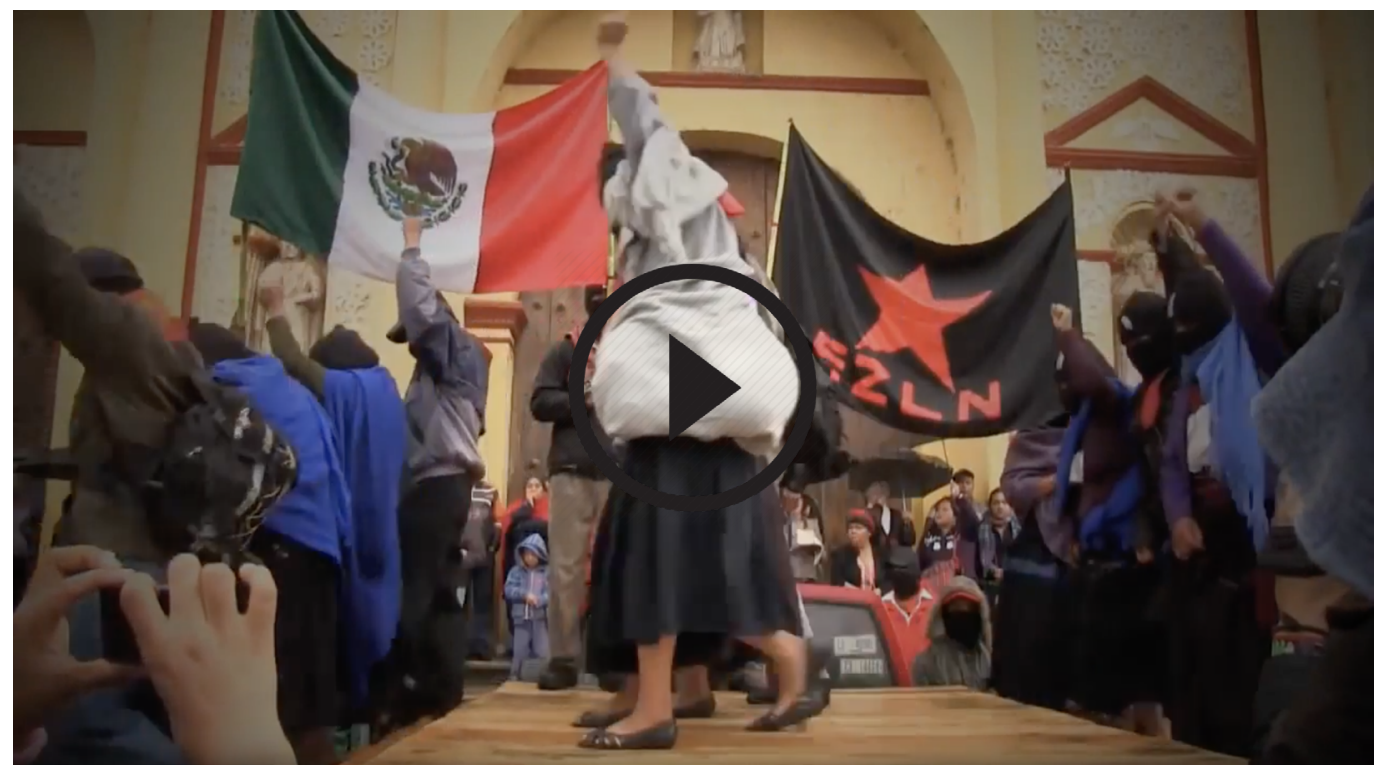

Dentro de esta continuidad de eventos del movimiento que también identifican autores como Arcos y Zagato (2017) o Aguirre Rojas (2017), posteriormente, en 2013 y 2014, se convocó en un llamado masivo para aprender de las y los zapatistas en lo que ellos denominaron como La Escuelita Zapatista, serie de eventos en los que se invitó a todos los miembros de la sociedad, simpatizantes con la causa del movimiento, a conocer de cerca cómo las comunidades indígenas ejercen nuevas formas de organización con base en sus 13 demandas colectivas: Democracia, justica, libertad, paz, salud, tierra, alimentación, trabajo, comunicación, independencia, cultura y educación; las mismas demandas por las que se levantaron en armas hace más de 20 años y que actualmente son una realidad gracias a la organización; lo que se hizo evidente no sólo fue la vigencia de esta lucha, sino la concepción compleja y transdisciplinaria que tienen las comunidades zapatistas de todas las dimensiones de la vida que deben estar en equilibrio para vivir dignamente.

Estos dos eventos — la marcha y la escuelita, el primero de naturaleza político-artística como una afrenta hacia los grupos en el poder y el segundo más enfocado hacia la educación desde donde se hizo una crítica hacia el privilegio exclusivo que se da al conocimiento que se produce desde las universidadesconstituyeron parte de los antecedentes de lo que para el 2016 y 2017 presentarían los y las zapatistas; es decir, otra serie de encuentros destinados a unir lo que en sus propias palabras "rescata lo mejor de la humanidad", esto es: las ciencias, las artes y los pueblos originarios. 


\title{
Los festivales zapatistas CompArte y ConCiencias por la Humanidad
}

\begin{abstract}
La política, la economía y la religión dividen, parcelan, parten. Las ciencias y las artes unen, hermanan, convierten las fronteras en ridículos puntos cartográficos. Pero, cierto, ni unas ni otras están exentas de la feroz división de clases y deben elegir: o contribuyen al mantenimiento y reproducción de la máquina, o contribuyen a mostrar su necesaria supresión. Como si en lugar de re-etiquetar la máquina, embelleciéndola o afinándola, el arte y la ciencia plantaran, sobre la cromada superficie del sistema, un letrero lacónico y definitorio: "CADUCO", "Tiempo Transcurrido", "para continuar viviendo deposite otro mundo".
\end{abstract}

Festival CompArte por la Humanidad, 2016. Presentación musical de las Bases de Apoyo Zapatistas, Caracol de Oventic, Chiapas, México. Fotografía: Francisco De Parres Gómez (Creative Commons)

Subcomandante Insurgente Galeano, 2016

Como se puede leer en este epígrafe de autoría del Subcomandante Insurgente Galeano (antes Marcos), en la actualidad el EZLN convoca a las ciencias y a las artes para construir alternativas en que todas y todos podamos vivir de una manera más justa, una posibilidad que sólo se puede ubicar, según Galeano y los zapatistas, "fuera de la máquina", es decir, fuera del sistema capitalista que se basa en la acumulación de la riqueza en manos de unos cuantos; por lo tanto, para buscar soluciones que trasciendan fronteras y que unan la complejidad de todos los conocimientos en beneficio de la humanidad, se convocó recientemente a los encuentros "CompArte por la Humanidad" y "L@s Zapatistas y las ConCiencias por la Humanidad" que tuvieron lugar en Chiapas, México, y que se extendieron a todo el mundo gracias los linderos virtuales del internet.

Estos encuentros, durante los dos años en que se han realizado, convoca-

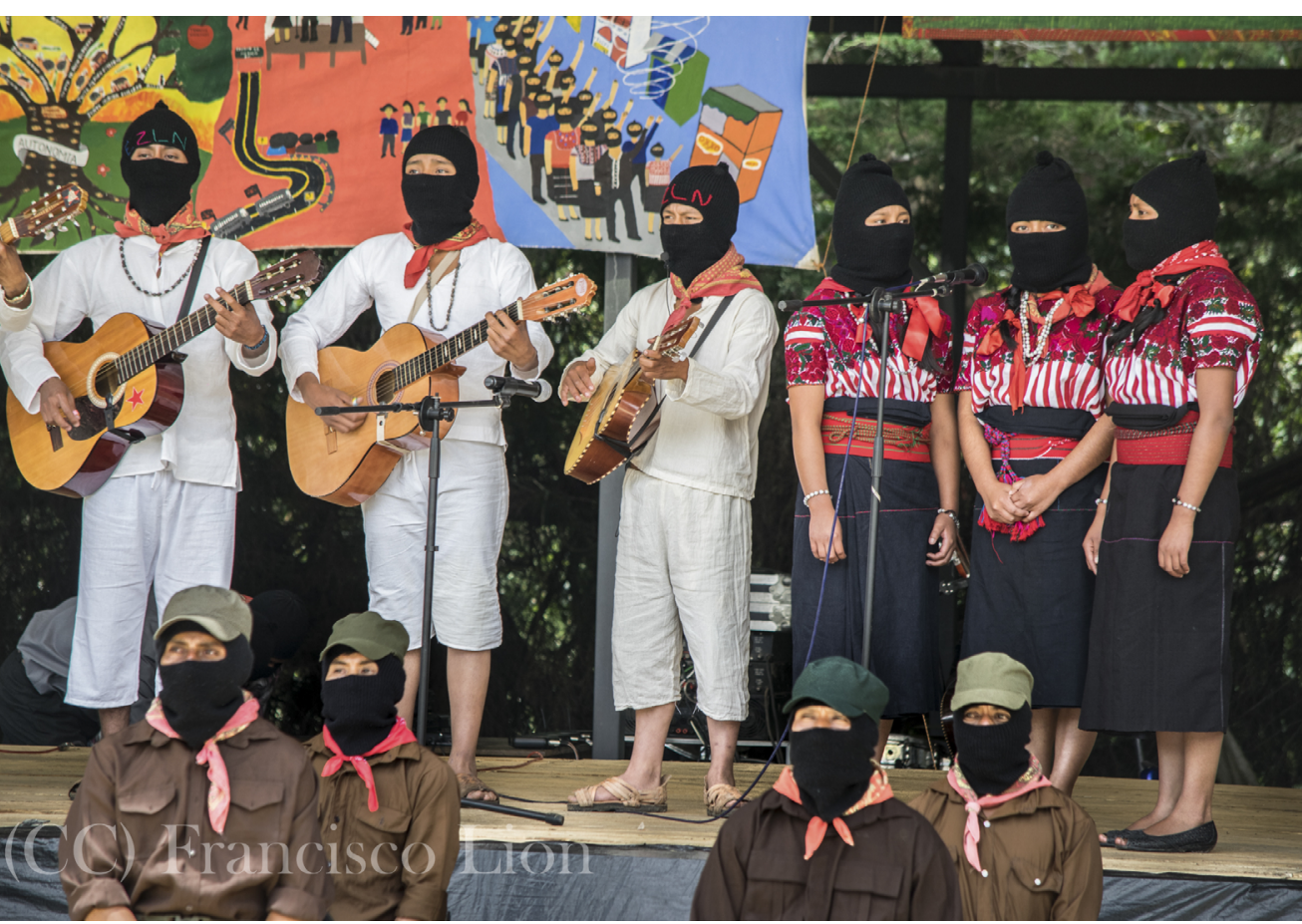
ron a miles de personas de diversos países, entre zapatistas, miembros de varios pueblos originarios, artistas, científicos y asistentes de todo el mundo, para conocer cómo es que las comunidades autónomas que han resistido al neoliberalismo trasnacional, plantean ahora las artes y las ciencias como ejes fundamentales que articulan sus demandas.

A continuación, recupero parte del comunicado que dio a conocer el EZLN en donde se invita a los artistas y científicos a participar, en el cual se hace evidente cómo es que las y los zapatistas conciben la continuidad entre las ciencias y las artes como elementos constitutivos de la resistencia: 
- Primero: que la grave crisis que sacude al mundo entero y que habrá de agudizarse, pone en riesgo la supervivencia del planeta y todo lo que lo puebla, incluyendo los seres humanos.

- Segundo: que la política de arriba no sólo es incapaz de idear y construir soluciones, también es una de las responsables directas de la catástrofe ya en curso.

- Tercero: que las ciencias y las artes son quienes rescatan lo mejor de la humanidad.

- Cuarto: que las ciencias y las artes representan ya la única oportunidad seria de construcción de un mundo más justo y racional.

- Quinto: que los pueblos originarios y quienes viven, resisten y luchan en los sótanos en todo el mundo son poseedores, entre otras, de una sabiduría fundamental: la de la supervivencia en condiciones adversas.

- Sexto:queelzapatismosigueapostando, enviday muerte, porla Humanidad.

(Subcomandante Insurgente Moisés, 2016a).

En relación con los artistas asistentes a los CompArte, simpatizantes con la causa, mas no miembros del EZLN, cabe señalar que, si bien no se trató de un acercamiento de carácter protocolario u "oficial", estos contaron con la asistencia de otros movimientos sociales en resistencia a nivel nacional e internacional, entre los que estuvieron los representantes del pueblo Mapuche de Argentina y Chile, así como miembros de las Black Panthers (Panteras Negras) de Estado Unidos, militantes del Movimiento Sin Tierra de los trabajadores de Brasil o los fundadores del Festival Artifariti, evento artístico que lucha por la liberación del pueblo Saharaui en África, por mencionar algunos. Además de personas en México que luchan contra los proyectos extractivistas como la minería, la tala de bosques o el fracking y demás actividades que están acabando con la naturaleza y desplazando a poblaciones enteras.

Entre las disciplinas artísticas que se apreciaron se contó con danza, pintura, teatro, performance, música, malabares, actos circenses, títeres, conferencias, muestras editoriales, fotográficas y de carteles, talleres de grabado, serigrafía, cuento, literatura, expresión corporal, escultura, poesía, entre muchas otras.

Por otra parte, los eventos ConCiencias contaron con la participación de científicos quienes discutieron temas como agroecología, astronomía, matemáticas, medicina, genética, saberes ancestrales, geofísica, inteligencia artificial, epidemiología, filosofía de la ciencia, geometría, geociencias, biología, biotecnología y astrofísica, por mencionar algunas.

Para citar un caso, en los temas relacionados con la naturaleza que tanto importan a los y las zapatistas, podemos ver que dentro de sus diferentes manifestaciones artísticas el maíz tiene un papel central, además de que se representan las comunidades cultivando la tierra como parte de sus trabajos colectivos, 
Festival ConCiencias por la Humanidad, 2017. Conferencias científicas para los Zapatistas, CIDECIUnitierra, San Cristóbal de las Casas, México. Fotografía: Francisco De Parres Gómez

(Creative Commons). en ese sentido, el arte funciona como transmisor de conocimientos, y en simultaneidad, en el área de las ciencias se discutió sobre las nuevas formas de cultivo y cómo estas benefician o afectan a la tierra, de tal manera que se articulan conocimientos que pueden provenir desde el arte o las nuevas tecnologías, pero en convivencia con los saberes ancestrales de los pueblos originarios que tienen un amplio conocimiento sobre estos temas, todo ello dirigido a abordar asuntos relacionados con la vida digna y cómo, por ejemplo, formas de producción poco éticas como los transgénicos están provocando tantas enfermedades.

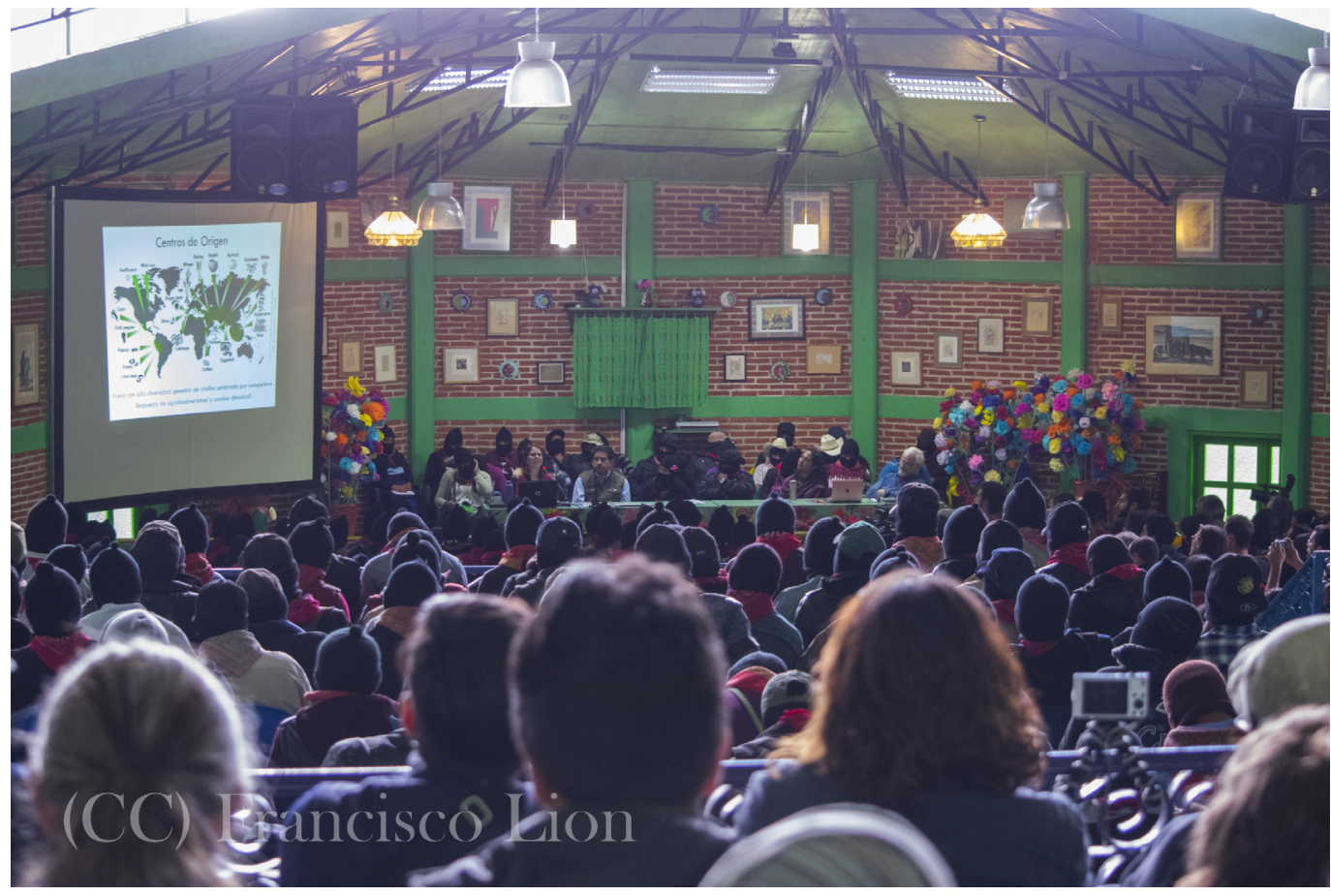

Así, desde una visión compleja y transdisciplinaria vemos como la naturaleza para los pueblos originarios además de ser fuente de subsistencia forma parte de su territorio, su cosmovisión y cultura, perspectivas que anteriormente en relación a la ciencia se podían considerar excluyentes u opuestas, sin embargo, aquí vemos posible cómo articularlas; a esta vinculación de varias perspectivas y dimensiones desde la complejidad le llamamos dialogicidad, en referencia a los diálogos que se establecen desde posiciones distintas.

Como se observa, estas experiencias organizadas por el EZLN en forma de festivales artísticos y encuentros científicos fueron fundamentalmente un amplio intercambio de conocimientos, donde se lograron establecer diálogos entre miembros del movimiento zapatista, líderes obreros, campesinos e indígenas, colectivos artísticos, estudiantes e intelectuales, grupos de científicos, así como cualquiera que se sintiera interpelado por el llamado a fortalecer la resistencia de la mano de las ciencias y las artes, y donde el objetivo fue y seguirá siendo la construcción de un mundo mejor fuera de la máquina: "Imagina..., imagina que 
todo lo que se gasta en política..., se dedicara a las ciencias y las artes. Que en lugar de campañas electorales y militares hubiera laboratorios, centros de investigación y divulgación científica, conciertos, exposiciones, festivales, librerías, bibliotecas, teatros, cines, y campos y calles donde reinaran las ciencias y las artes, y no las máquinas." (Subcomandante Insurgente Galeano, 2016).

\section{Reflexiones finales “¿y tú, qué?..."}

Festival ConCiencias por la Humanidad, 2017. Palabras del Subcomandante Insurgente Galeano, CIDECIUnitierra, San Cristóbal de las Casas, México. Fotografía: Francisco De Parres Gómez (Creative Commons).
A ustedes, la juventud consciente, a los creadores y multiplicadores de artes y ciencias, los reconocemos como una gran luz en medio de tanta muerte y oscuridad, los necesitamos para seguir soñando, luchando y haciendo cada vez más grande eso que los poderosos tanto temen y que se llama democracia, libertad y justicia.

María de Jesús Patricio Martínez, Vocera del Concejo Indígena de Gobierno, 2017

Como se pudo apreciar de manera breve al inicio del texto, anteriormente existía una visión de la ciencia que para entender el mundo fragmentaba y simplificaba el conocimiento del mismo, además de que ésta se encontraba reservada para grupos de élite donde la población a nivel más general se encontraba excluida. Posteriormente, en el siglo XX y como una de las propuestas más actuales, surgieron nuevos caminos con un posicionamiento ético que plantearon que es preciso construir puentes entre el mayor número posible de variables que den cuenta de la complejidad y transdisciplinariedad de la vida misma, donde es necesario incluir no sólo lo que se produce desde la academia sino además el conocimiento propio de la humanidad global integrando de manera igualitaria a los pueblos originarios.

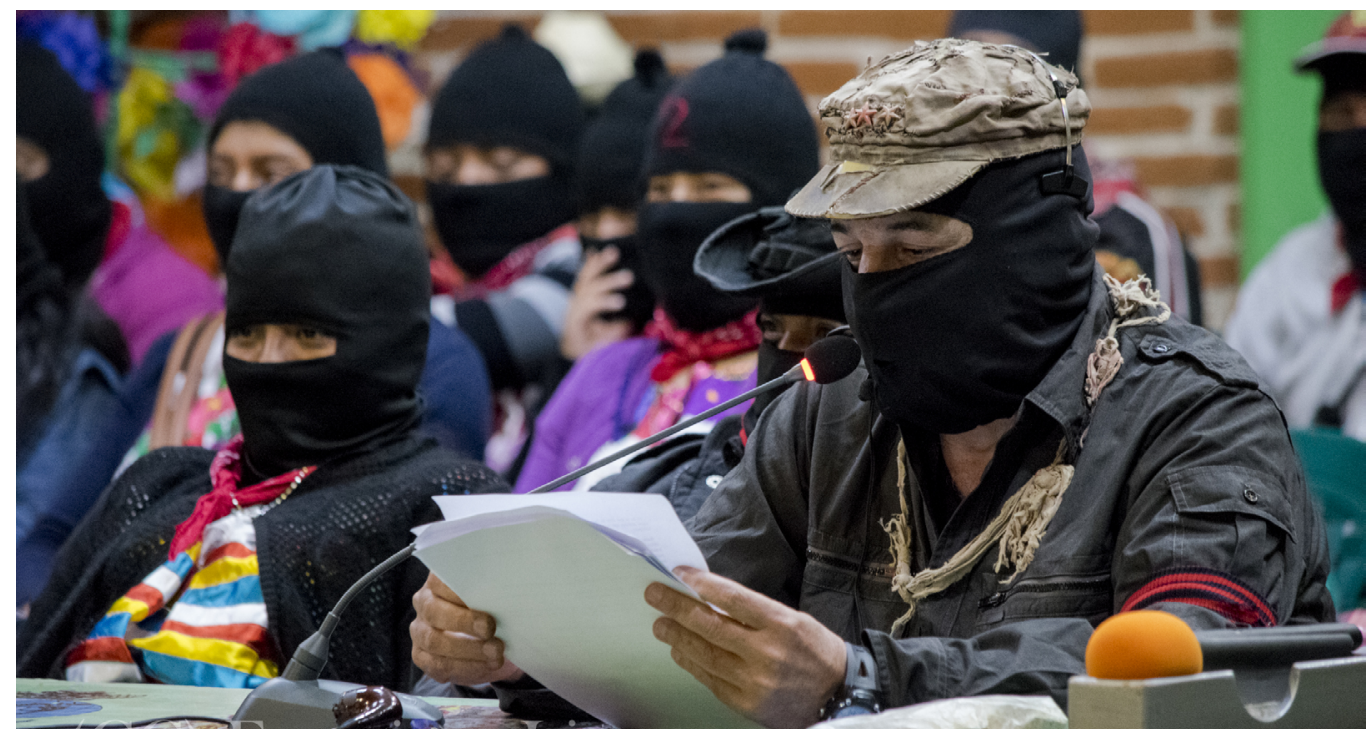


Para finalizar, abordamos el ejemplo de cómo el Ejército Zapatista de Liberación Nacional hace posible esto; quienes en su práctica conciben múltiples factores de la vida que tienen que ser garantizados para todos y con ello construir un mundo nuevo; o como dicen ellos: una "casa nueva" más justa, digna y equilibrada, encontrando las continuidades que recorren desde las ciencias hasta las artes de manera compleja y transdisciplinaria.

Es necesario decir también que la tarea de escapar de la crisis que cada vez se agudiza más y construir una nueva sociedad nos involucra a todas y a todos, no será posible hacerlo de otra manera ya que desde una posición compleja y transdisciplinaria cada ser humano que busque una solución ética tiene algo que aportar.

Para concluir, nos quedamos con esta invitación que nos hacen los compañeros y compañeras zapatistas en voz de los Subcomandantes Insurgentes Moisés y Galeano para reflexionar... ya tocará a cada uno de nosotros si atendemos a su llamado:

A nosotros nos toca primero saber que esa casa es posible y necesaria. Y luego, pues bueno, lo más fácil: nos toca construirla. Y para eso necesitamos el saber, el sentir, la imaginación, necesitamos las ciencias y las artes. Necesitamos otros corazones. Ya llegará el día en que nos encontremos con quienes hacen las artes y las ciencias. Ese día les daremos un abrazo y, como bienvenida, les recibiremos con una sola pregunta: "¿Y tú qué?" (Subcomandante Insurgente Moisés, Subcomandante Insurgente Galeano 2016a).

\section{Referencias}

- Aguirre, C. (2017). Un Arte que se CompArte. Dossier Contrahistorias. Pensamiento Crítico y Contracultura. Núm. 27, Marzo-Agosto 2017. México: Jiménez Servicios Editoriales. Pp. 1-118.

- Amozurrutia, J. (2012). Complejidad y sistemas sociales. Un modelo adaptativo para la investigación interdisciplinaria. Universidad Nacional Autónoma de México, México. Centro de Investigaciones Interdisciplinarias en Ciencias y Humanidades.

- Arcos, N. y Zagato, A. (2017). El Festival "Comparte por la Humanidad". Estéticas y poéticas de la rebeldía en el movimiento Zapatista. Argentina: Páginas, Revista digital de la Escuela de Historia, Universidad Nacional de Rosario, Año 9, Núm, 21. Pp. 75-101.

* Carrizo, L., Espina, M. y Klein, J. (2003). Transdisciplinariedad y Complejidad en el Análisis Social, Gestión de Transformaciones Sociales (MOST). Organización de las Naciones Unidas para la Educación, la Ciencia y la Cultura.

* Castellanos, A. (2015). "Zapatismo y horizonte de futuro". En EZLN, El Pensamiento Crítico Frente a la Hidra Capitalista II. México: Ejercito Zapatista de Liberación Nacional, Pp. 113-126. 
* Descartes, R. (1942). Discurso del método y meditaciones metafísicas. (Manuel G. Morente, trad.) España: Espasa - Calpe S.A. (Obra original publicada en 1637).

* EZLN. (2015). El Pensamiento Crítico Frente a la Hidra Capitalista I. Participación de la Comisión Sexta del EZLN. México: Ejercito Zapatista de Liberación Nacional.

* Fernández, M. (2013). Zapatismo o barbarie. Apuntes sobre el movimiento zapatista chiapaneco. Argentina: Fundíbulo Ediciones.

* Gascón, P., Rosario, M. y Vizcarra, I. (Coords.) (2014). Reflexiones en torno a la complejidad y la transdisciplina. México: Universidad Autónoma Metropolitana, Universidad Autónoma del Estado de México.

* González Casanova, P. (2015). "Crisis terminal del capitalismo o crisis terminal de la humanidad". En EZLN, El Pensamiento Crítico Frente a la Hidra Capitalista II. México: Ejercito Zapatista de Liberación Nacional, Pp. 285-296.

* Morin, E. (1997). Introducción al pensamiento complejo. Editorial Gedisa, Barcelona.

* Nicolescu, B. (1994). La Transdisciplinariedad. Manifiesto. Portugal: Ediciones Du Rocher.

- López y Rivas, G. (2014). Autonomía de los pueblos indios y zapatismo en México. China: Ocean Sur, Ocean Press.

* Patricio, M. J. (2017) Discurso pronunciado por María de Jesús Patricio Martínez, Vocera del Consejo Nacional Indígena en la Universidad Nacional Autónoma de México. 28 de noviembre de 2017, Ciudad Universitaria. (Disponible en: https://zapateando. wordpress.com/2017/11/28/discurso-de-maria-de-jesus-patricio martinez-vocera-del-cig-en-ciudad-universitaria-28-de-noviembre-de-2017/\#respond)

* Subcomandante Insurgente Galeano. (2016). Las artes, las ciencias, los pueblos originarios y los sótanos del mundo. México: Chiapas, Comité Clandestino Revolucionario Indígena, Comandancia General del EZLN, Enlace Zapatista. (Disponible en: http://enlacezapatista.ezln.org.mx/2016/02/28/las-artes-las-ciencias-los-pueblos originarios y-los-sotanos-del-mundo/)

* Subcomandante Insurgente Moisés. (2016a). Convocatoria zapatista a actividades 2016. México: Chiapas, Comité Clandestino Revolucionario Indígena, Comandancia General del EZLN, Enlace Zapatista. (Disponible en: http://enlacezapatista. ezln.org.mx/2016/02/29/convocatoria-zapatista-a-actividades-2016/)

* Subcomandante Insurgente Moisés (2016b). El arte que no se ve ni se escucha. México: Chiapas, Comité Clandestino Revolucionario Indígena, Comandancia General del EZLN, Enlace Zapatista. (Disponible en: http://enlacezapatista.ezln.org. mx/2016/08/03/el-arte-que-no-se-ve-ni-se-escucha/ )

* Subcomandante Insurgente Galeano y Subcomandante Insurgente Moisés. (2016a). Las Artes y las Ciencias en la historia del (neo) Zapatismo. México: Chiapas, Comité Clandestino Revolucionario Indígena, Comandancia General del EZLN, Enlace Zapatista. (Disponible en: http://enlacezapatista.ezln.org.mx/2016/12/28/ las-artes-y-las-ciencias-en-la-historia-del-neo-zapatismo/) 
* Subcomandante Insurgente Galeano y Subcomandante Insurgente Moisés (2016b). Una casa, otros mundos. México: Chiapas, Comité Clandestino Revolucionario Indígena, Comandancia General del EZLN, Enlace Zapatista. (Disponible en: http://enlacezapatista.ezln.org.mx/2016/09/12/una-casa-otros-mundos/ )

* Velilla, M. (Compilador), (2002). Manual de iniciación pedagógica al Pensamiento Complejo, Corporación para el Desarrollo Complexus, Instituto Colombiano de fomento a la Educación Superior.

* Wallerstein, I. (2015). "Los movimientos antisistémicos y el futuro del capitalismo". En EZLN, El Pensamiento Crítico Frente a la Hidra Capitalista II. México: Ejercito Zapatista de Liberación Nacional, Pp. 264-284.

\section{Cómo citar este artículo}

* De Parres Gómez, F. (2018). Continuidad entre la ciencia y el arte en el movimiento Zapatista: una mirada desde la complejidad. Revista Digital Universitaria (RDU). Vol. 19, núm. 2 marzo-abril. DOI: http://doi.org/10.22201/codeic.16076079e.2018. v19n2.a3 